\title{
Argon laser suture lysis with different suture materials. An experimental study
}

\author{
C E Hugkulstone, A F Spencer, S A Vernon
}

\begin{abstract}
In an in vitro study, 10/0 nylon was found to require a significantly lower laser energy density to produce suture lysis following a single shot than either 10/0 Dacron or 10/0 prolene. Nylon and Dacron monofilament sutures ruptured at reproducible energy levels without significant observable changes at subthreshold irradiation. Prolene, however, was observed to stretch at energy levels below the lysis threshold, under the standard tension produced by a weight of $0.36($ SD 0.02$) \mathrm{g}$. This feature may be of value when performing laser treatment to trabeculectomy flap sutures in the early postoperative period.

(Br f Ophthalmol 1994; 78: 390-391)
\end{abstract}

In 1984, Hoskins and Migliazzo reported the technique of cutting the scleral flap sutures of a trabeculectomy with the argon laser, as an aid to rescue failing blebs.

Shortly afterwards, Savage and colleagues described the use of this technique in an attempt to obtain the early postoperative safety of trabeculectomy plus the long term intraocular pressure control offered by full thickness drainage surgery. ${ }^{23}$ In addition, to reduce further the incidence of a shallow or flat anterior chamber following trabeculectomy, tight closure of the scleral flap with subsequent laser suture lysis has been proposed. ${ }^{34}$

In these reports, the suture material employed was nylon and of $10 / 0$ gauge in the majority of cases. ${ }^{1-3}$ Other 10/0 monofilament suture materials have been introduced since this technique was first described, such as Dacron and prolene. To determine if any difference existed in the levels of laser energy required to produce suture lysis, an in vitro study was performed on three suture materials.

\section{Methods}

Three different suture materials (10/0 nylon, 10/0 Dacron, and 10/0 prolene) were obtained from the same manufacturer. Multiple lengths of these sutures were suspended against a uniform matt white background, under a constant light tension produced by an attached weight of 0.36 (SD 0.02) g. A calibrated HGM PC argon laser was employed, using the green output (wavelength $514 \mathrm{~nm}$ ).

The laser was focused using the slit-lamp and single shots were applied directly to the sutures, without the use of a contact lens. Only one shot was applied to each suture length, to avoid localised variation in tension from previous subthreshold applications. The laser energy was increased in a stepwise manner from a random- ised starting point, and the experienced laser operator (SAV) was masked to the power settings. It was not possible to mask the actual suture material, because of the different colours of the sutures (nylon, black; Dacron, green; and prolene, blue).

In a pilot study, we found that using the reported laser parameters for suture lysis in a clinical situation $^{1-4}$ (a shot size of $50 \mu \mathrm{m}$ and a duration of $0 \cdot 1$ second) caused lysis of all three materials with the power set at the minimum possible value (0.04 W for our laser), even when using the green output, which reduces the power by $30 \%$ from that displayed. Lysis still occurred at the minimum power setting when the duration was reduced to 0.05 seconds. The spot size was then increased progressively until lysis occurred with the power setting in the middle of the available range, to allow comparison of the laser powers necessary for suture lysis. This was found to occur with a spot size of $500 \mu \mathrm{m}$. These settings were constant in the study, the energy delivered being varied by the power output alone.

The first stage with each suture material was designed to identify the approximate power setting at which suture lysis occurred. The power was started from a random value between 0.1 and $0.5 \mathrm{~W}$, and was increased in steps of $0.1 \mathrm{~W}$. This was repeated five times for each suture, and the model value at which instantaneous suture lysis occurred following a single shot was calculated. The second stage was intended to identify the mean power required for lysis of the three suture materials, to the nearest $0.01 \mathrm{~W}$. For this stage, the statistical mode of the first stage was reduced by $0 \cdot 1 \mathrm{~W}$. This power setting was then increased by $0.01 \mathrm{~W}$ steps, starting randomly between 0.00 and $0.03 \mathrm{~W}$. This was again repeated five times for each suture, the value at which lysis occurred after a single shot was again noted, and the mean (SD) calculated.

Statistical analysis was with the MannWhitney test.

\section{Results}

The values at which instantaneous suture lysis following a single shot occurred, in both stages 1

Table 1 Power settings (W) for suture lysis of 1010 nylon and 1010 Dacron (0.05 second duration)

\begin{tabular}{|c|c|c|c|}
\hline \multicolumn{2}{|l|}{ Nylon } & \multicolumn{2}{|l|}{ Dacron } \\
\hline Stage 1 & Stage 2 & Stage 1 & Stage 2 \\
\hline $\begin{array}{l}0.4 \\
0 \cdot 4 \\
0 \cdot 4 \\
0 \cdot 4 \\
0.4\end{array}$ & $\begin{array}{l}0.35 \\
0.35 \\
0.35 \\
0.33 \\
0.33\end{array}$ & $\begin{array}{l}1 \cdot 1 \\
1 \cdot 2 \\
1.2 \\
1 \cdot 2 \\
1 \cdot 3\end{array}$ & $\begin{array}{l}1.13 \\
1.12 \\
1.11 \\
1 \cdot 16 \\
1.11\end{array}$ \\
\hline
\end{tabular}




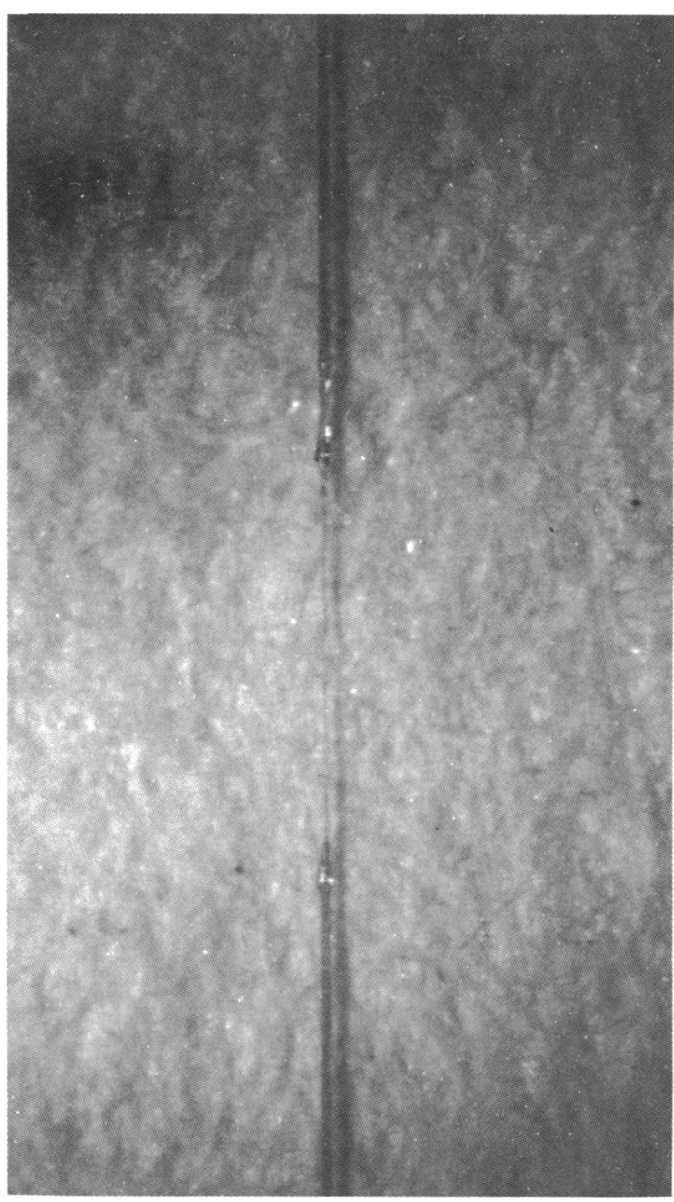

Figure $110 / 0$ prolene suture showing marked thinning after a single subthreshold laser application.

and 2, are shown in Table 1 for nylon and Dacron. In stage 1 , the modal value for nylon was $0.4 \mathrm{~W}$, and for Dacron $1.2 \mathrm{~W}$. After stage 2, the mean value for nylon was significantly lower than that for Dacron $(0 \cdot 34(0 \cdot 01) \mathrm{W}$ versus $1 \cdot 13$ $(0.02) \mathrm{W}$ respectively, $\mathrm{p}<0.01)$.

For the prolene suture, the results were quite different. Lysis did not occur in three of the five sequences within the power limits of the laser using the experimental parameters. In the other two sequences, lysis did occur, both at $1.2 \mathrm{~W}$. Thus, the modal value for stage 1 was 'no lysis', and so stage 2 was not performed. However, marked stretching and thinning occurred at the site of the laser burn (Figure 1) in all five sequences, over a power range of $1 \cdot 0-1 \cdot 4 \mathrm{~W}$. This effect was not seen with the other two suture materials, where subthreshold energies produced little visible effect. To produce lysis of the prolene suture, a duration of $0 \cdot 1$ second had to be employed, and the results are shown in Table 2. With this longer duration, stretching of the suture was only rarely observed.

To compare the three suture materials for ease

Table 2 Power settings prolene $(0 \cdot 1$ second duration $)$

\begin{tabular}{ll}
\hline Stage 1 & Stage 2 \\
\hline 1.0 & 0.90 \\
0.9 & 0.92 \\
0.9 & $0 \cdot 86$ \\
1.0 & 0.85 \\
0.9 & 0.83 \\
\hline
\end{tabular}

Mode $=0.9$.

Mean $=0.87(0 \cdot 04)$ of laser suture lysis, the mean energy densities (MEDs, in $\mathrm{J} / \mathrm{mm}^{2}$ ) were calculated, from power setting $\times$ duration/area of spot size, based on the values obtained in stage 2 . The MED for nylon $(0.087(0.003))$ was significantly lower than that of both Dacron $(0.287(0.005), \mathrm{p}<0.1)$ and prolene $(0.444(0.019), \mathrm{p}<0.01)$. Prolene also required a significantly higher MED than Dacron $(\mathrm{p}<0.01)$.

\section{Discussion}

The major difference between this experimental study and the results of clinical work is in the size and duration of the burn required to produce suture lysis. A number of factors may be involved. The higher energy required for lysis in the clinical setting may be related to access to the suture, with conjunctiva attenuating the laser beam. In one study using $9 / 0$ nylon, the average power required for lysis was $0.7 \mathrm{~W}$, with a duration of 0.1 second and requiring two applications per suture. ${ }^{4}$ Thus, the average MED per shot was $35 \mathrm{~J} / \mathrm{mm}^{2}$, much higher than the value found for 10/0 nylon in the current study. Three of the four case reports in Savage and Simmons' original paper ${ }^{2}$ also required a higher MED (of $50 \mathrm{~J} / \mathrm{mm}^{2}$ ) to lyse $10 / 0$ nylon sutures. Both studies used a spot size of $50 \mu \mathrm{m}$. Clinically, the tension that the suture is under will vary, dependent on both the surgeon and the contact lens technique. In addition, in vivo the suture material has been exposed to extracellular fluid, and this may play a role in altering the energy needed to produce lysis. However, the aim of our study was to identify any inherent differences between the suture materials under constant conditions, rather than to mimic the clinical situation.

Although the size of the laser burn was different from that employed clinically, it was constant throughout this study. The pulse duration had to be doubled to lyse the prolene suture and this is reflected in the higher MED required. Nylon had the lowest MED, presumably by virtue of its colour (black) and so its absorption of laser light energy was highest.

However, the most interesting finding was the thinning observed with prolene. If this property of $10 / 0$ prolene can be utilised in vivo, it should become possible to titrate the reduction in outflow resistance during postoperative laser treatment of the sutures supporting the scleral flap. A similar effect has been attempted by the placement of multiple 10/0 nylon flap sutures and selective suture lysis. ${ }^{34}$ Both this and the technique employing a removable suture, ${ }^{5}$ run the risk of a sudden decrease in outflow resistance leading to shallow or flat anterior chambers and extensive drainage blebs. Judicious use of the laser with $10 / 0$ prolene may reduce this possibility.

Further studies are required to ascertain the effect of exposure to tissue fluid on laser lysis threshold energy levels, and to determine if the properties observed with $10 / 0$ prolene in this study can be reproduced in the clinical setting.

Our thanks are due to Uniplex for kindly supplying the suture materials. None of the authors have any proprietary interest in this company or their products.

1 Hoskins HD Jr, Migliazzo C. Management of failing filtering blebs with the argon laser. Ophthalmic Surg 1984; 15: 731-3. with planned early conversion from scleral flap to fullthickness operation using argon laser. Ophthalmic Laser Ther 1986; $1: 201-10$.

3 Savage JA, Condon GP, Lytle RA, Simmons RJ. Laser suture lysis after trabeculectomy. Ophthalmology 1988; 95: 1631-8.

4 Melamed S, Ashkenazi I, Glovinski J, Blumenthal M. Tight scleral flap trabeculectomy with postoperative laser suture lysis. Am f Ophthalmol 1990; 109: 303-9.

5 Johnstone MA, Wellington DP, Zeil CJ. A releasable scleralflap tamponade suture for guarded filtration surgery. Arch Ophthalmol 1993; 111: 398-403. 